\title{
Grand challenges in low-temperature plasma physics
}

\section{Christine Charles*}

Space Plasma, Power and Propulsion Laboratory, Research School of Physics and Engineering, The Australian National University, Canberra, ACT, Australia

*Correspondence: christine.charles@anu.edu.au

Edited by:

Antonio D'Angola, Università della Basilicata, Italy

Reviewed by:

Antonio D'Angola, Università della Basilicata, Italy

Agnes Granier, Centre National de la Recherche Scientifique, France

Keywords: low-temperature plasmas, space plasmas, laboratory plasmas, industrial plasmas, plasma-surface interactions

\section{INTRODUCTION}

A plasma is a hot ionized gas and classification of this fourth state of matter can be initially done using the basic concept of temperature. A prime example is the Sun which exhibits a very hot plasma in its core with temperatures of about $1.5 \times 10^{7} \mathrm{~K}$ (a result of fusion reactions with a proton density of $\sim 10^{26}$ $\mathrm{cm}^{-3}$ ) and cooler surface temperatures of about $6000 \mathrm{~K}$ [1]: the Solar wind originates from the Solar Corona, expands into the universe and impacts the Earth' magnetosphere and ionosphere, the two plasma layers surrounding Earth's gaseous atmosphere. Aurorae in the Northern and Southern skies near the magnetic poles are examples of this interaction. The electron density in the ionosphere is low $\left(10^{4}-10^{6}\right.$ $\mathrm{cm}^{-3}$ ) and the background neutral gas density is also low (about $10^{8} \mathrm{~cm}^{-3}$ or $3 \times$ $10^{9}$ Torr at $300 \mathrm{~km}$ altitude) approaching the "space-like" environment created in laboratories to develop and test hardware for space use (i.e., satellites and payloads). At the surface of the Earth lightning strikes of a storm are naturally occurring plasmas operating near atmospheric pressure (neutral density of about $2 \times 10^{19} \mathrm{~cm}^{-3}$ ) with large electron densities (about $10^{15}$ $10^{17} \mathrm{~cm}^{-3}$ ) characteristic of streamers, arcs and filamentary discharges. The temperatures and densities of neutral and charged particles are critical parameters affecting the physical mechanisms within the plasma such as particle transport and collisional processes [2] and their range spans many orders of magnitude, opening doors to an extremely wide range of plasma applications.

Plasmas in which fusion reactions take place are often referred to as "hot" plasmas. The high-temperature plasma community has a well-defined aim of triggering and controlling fusion plasmas (as can be found in the Sun's core) for energy production, with various worldwide large scale programs or experiments in place (i.e., the International Thermonuclear Experimental Reactor ITER, the National Ignition Facility ICF...) [3]. Hot plasmas in space also include relativistic plasmas (highest electron temperatures) and quantum plasmas (highest electron densities). All other plasmas are classified as low-temperature or "cold" plasmas: those gaseous plasmas or electrical discharges have been successfully harnessed and studied in the laboratory since the 1920s and in space using satellites since the 1960s. The latter two plasma examples essentially sit at the opposite ends of the "cold" plasma spectrum. The wide range of available plasma parameters has largely contributed to the long and expanding list of plasma applications as a result of both scientific and economic drivers: the temperature range of the neutral and/or ionized species allow heat and particle control to burn, melt, cut, coat, grow materials from the macroscopic to the micro- and nanoscale via "so called" plasma processes.

Amongst thousands of plasma applications processes, a few examples are presented to show the past, present and future key role cold plasma physics must play in addressing the challenges facing the modern world: predicted energy crisis, environmental issues and ecosystems, global climate variation, population growth and biomedical concerns. In addressing those issues we must better understand the physics of the atmosphere, ionosphere and magnetosphere, the physics of the plasma interacting with a boundary and develop new clean sources of energy.

\section{SPACE AND LABORATORY PLASMAS}

Successful operation of satellites in Low Earth Orbits within the ionosphere (the inner plasma layer in the Earth's atmosphere) plays a key role not only in international global security but also in climate change and weather monitoring. In addition to space weather related communications glitches (from temporal to catastrophic failure) there is a growing and fierce international competition for access to "premium" Earth orbits especially geostationary, and increasing concern for collisions with other satellites or space debris. It takes 2 years to build a large telecommunication satellite for a "commercially guaranteed" lifetime of 15 years, interestingly similar to the slowest dynamic scale of solar activity, defined as the solar cycle. However, solar processes with shorter time scales are largely responsible for day to day variation of space weather with a reported link between solar geomagnetic variation, solar-wind velocity and solar-wind energy density [4].

The energy stored in the dynamical behavior of the Sun's complex magnetic field structures is occasionally released in dramatic disruptions (Coronal Mass Ejections) producing large space storms in the magnetosphere of the Earth and investigated in the laboratory for nearly a century in "Terrella experiments" $[5,6]$. These storms can have major consequences for communications and impact almost every aspect of our life through telecommunications, telephones or when simply driving to the shops using a Global Positioning System (GPS). It also plays a 
major role in securing many countries' defense as long-range radars are significantly perturbed by variations in space weather [4, 7].

A surprising and unresolved mystery of the cosmos is the spectroscopically measured acceleration (over a short distance of about $300 \mathrm{~km}$ within the low density solar corona) of the electrically neutral solar wind plasma to proton temperatures of up to $1-1.5 \times 10^{6} \mathrm{~K}$, approaching that of the Sun's core $[1,8]$. Particle momentum is transported outward via the expanding and decreasing density solar wind into the solar system and beyond. This high velocity outward flux of particles yields a hot and tenuous solar wind and most importantly contains a complex frozen-in magnetic field structure creating the Parker Spiral. It is this magnetic field which connects with that of the Earth, in the bow shock of the magnetosphere above the ionosphere. Since the mass loss due to the solar wind can be considered negligible, the life cycle of the Sun and other similar rotating stars are quite well determined from the initial Big Bang to their death as Red Giants (before their final collapse). In strong contrast to the successful predictive models of the Sun's sequential phases, only general models (or guesses more accurately) have been proposed to explain coronal heating but even now, after more than 40 years of intensive research, there is no agreement amongst scientists as to how mass and energy flow to the corona.

Interestingly, the main parameter ranges of the solar corona (electron and ion densities, temperatures and magnetic field strength) can be experimentally created in terrestrial laboratory plasmas, although we have to recognize that a spatial scaling about six orders down is necessary [9]. Large thermal vacuum chambers are being developed worldwide for development and testing of hardware for spacecrafts and this should open door to a range of experimental campaigns aimed at providing new data relevant to charged particle transport and heating processes. Acceleration of the solar wind remains a great challenge which may be answered by judicious acquisition and analysis of laboratory data combined with space data (from instruments carried on satellites) and perhaps from a new approach to experimental physics carried out in space to mitigate the scaling issues. Observational constraints exist both in the laboratory and in space and in a number of cases, the body of information is not yet sufficient to yield adequate data interpretation in phase with a theoretical understanding based on mathematical laws of physics. Aside from this example, there are many more unresolved phenomena with no clear consensus such as plasma detachment from a magnetic field, magnetic reconnection, cross-field diffusion, collision-less shocks, wave particle interaction [1,9-11].

Perhaps these research areas could be grouped as plasma challenges in which the boundary is a gas, a discontinuity (shock) with the plasma or another plasma. It includes the topic of plasma thrusters, a "laboratory plasma" expanding in the ionosphere. Most of the areas reviewed below focus on the interaction between a plasma and a solid or liquid boundary.

\section{HIGH-TECHNOLOGY INDUSTRY}

The interaction between a reactive or nonreactive laboratory plasma and any solid or liquid surface is complex and consequently offers an extremely wide range of applications [12]. Nowadays many challenges reside in the cross-disciplinary nature of each development and include aspects of physics, chemistry, materials science, biology and engineering. The parameters are numerous, often non-linearly coupled and predictive models are usually not available [11, 13]. Hence many achievements have been obtained by empirical means first and subsequently developed and improved by the use of models and computer simulations.

The development of "plasma" or "dry" processes for the miniaturization of integrated circuits (ICs) from the 1970s has revolutionized the high-tech microelectronics industry based on the invention of the transistor in 1947. It typically takes 2 months and about thirty plasma processes for the manufacturing in a fabrication line of a semi-conductor IC: such processes include material etching and deposition of metals, insulators and semi-conductors. Over the past 40 years, plasma technology has reduced the size of electronic components by a factor of 250,000 while allowing processes over increasingly large wafers. Highest quality features and lowest price are now key drivers for access to laptop computers, mobiles phones, digital cameras and GPS systems with nearly all industries having efficient and reliable computerized control systems to provide their services. Here low pressure plasmas (a few orders of magnitude lower than atmospheric pressure) not in thermal equilibrium (the gas temperature is much lower than the electron temperature) are commonly used which require the use of expensive vacuum systems and clean rooms, the use of highly reactive and often toxic gases necessitating safety equipment and procedures and the use of state of the art precision tools and materials bulk or surface diagnostics [14].

Current challenges of the microelectronics industry include the move from 300 to $450 \mathrm{~mm}$ in diameter silicon wafers, the development of three-dimensional silicon-based integrated circuits (a first approach is the integration of two "flipped" wafers necessitating "Through Silicon Vias," dielectric etching with large aspect ratios, extended mask lifetime), integrated opto-electronics (merging of III-V and silicon semi-conductor technologies), further development and integration of new materials in communications devices (quantum transistors and nanotechnology) or in MicroElectroMechanical Systems (MEMS) or more recently in BioMEMS.

Beam extraction from plasmas include neutral beam injection in fusion experiments, ion beam extraction (from low to high energy) and acceleration, and electron beams used as neutralizers in electric propulsion in space (i.e., ion gridded and Hall effect thrusters). Focused ion beams are key diagnostics tools for reverse engineering, materials science and the emerging field of nuclear forensic. Many challenges related to the development of ICs such as process uniformity and substrate charging are also relevant to thin film coatings for aerospace applications or even the control of electrical charging of satellites. The inter-connectivity between applications is an exciting aspect of cold plasma physics and engineering. Similarly, impressive advances in laserdriven plasma accelerators have yield the generation of very high energy electron $(\mathrm{MeV}-\mathrm{GeV})$, proton $(\mathrm{MeV})$ and $\mathrm{X}$-ray beams $[15,16]$. For example, with plasma 
acceleration stages that are only a few centimeters long, acceleration several orders of magnitude greater than that generated by conventional accelerators is achieved and produces particle energies comparable to those offered by synchrotron light sources. Recent developments indicate that these systems will play an important role in future investigation of matter in particular fundamental short timescale events or transient dynamics relevant to biological, chemical and solid-state "targets." Practical applications will strongly benefit from the design and construction of compact accelerators.

\section{RESOURCES ON EARTH}

Human activity on Earth over the past two centuries and human space use over the past 55 years have expanded by using non-renewable supplies of fossil fuels with a large hydrocarbon content (coal, petroleum, natural gas): initially with coal and steam engines, then with petrol and combustion engines, and also with electricity (which can be produced in various ways). The fixed supply of hydrocarbon fuels cannot indefinitely feed the population growth and individual energy needs. Deforestation, soil erosion, ocean pollution on Earth, carbon dioxide emission in the Earth' atmosphere and space debris orbiting the Earth increasing affect the Earth's fragile ecosystems and equilibrium. Can low-temperature plasmas contribute to a better transport, storage and management of our energy resources, and to a better control of waste products on Earth and in space? The diversity of energy-related plasma applications implies that the fourth state of matter will be a key player in sustainability and waste management.

An iconic example is the evolution of lighting devices: at night and from space, Earth appears as an illuminated sphere and the energy consumption related to lighting is significant. One of the cheapest plasma devices making direct use of the photons in the plasma is the fluorescent tube, essentially a plasma lamp containing neon gas with a small amount of mercury vapor confined between electrodes at both ends. It has taken two decades for this technology to mature and provide a good electrical to light conversion efficiency and new developments aiming at moving away from the use of mercury while maintaining good conversion efficiency are proving difficult [12].

Power generation from fusion reactors is still a long way off and the inclusion of renewable energy (solar, wind) into the grid creates a set of new challenges. A large fraction of the population in developing countries has no access to modern affordable energy services and small scale systems can provide access to "offgrid" energy: solar technology, fuel cell technology, wind technology and perhaps artificial photosynthesis in the future. Could the latter produce a sustainable global hydrogen economy in the developing world? Plasma technology usually finds its way in most applications and an important challenge will be to take into account the "real" net energy cost and environmental impact.

Electric arcs, plasma jets or torches are very widely used in the industry and these are "thermal" plasmas operating at or near atmospheric pressure. Both the electrons and heavy species are at high temperature and the intense heat of the arc is used to melt and fuse the metals edges (arc welding) or to cut through metals (plasma cutting). Electric arc furnaces are used to produce metals such as steel. The intense heat of the arc is increasingly used for waste treatment (from household garbage to highlytoxic chemicals) to convert organic liquids, ozone-depleting substances and fluorocarbon greenhouse gases into harmless substances. In some cases useful products, such as syngas (a mixture of hydrogen and carbon monoxide), can be combusted to produce electricity. Overall the process may be costly and not energy efficient but can solve important problems. Wear- or thermally-resistant coatings (the coating of jet engine turbine blades with zirconia) can be sprayed by injected particles into the plasma jet where they partially melt before being blasted onto the surface. Plasma spraying can be reduced down to the nano-scale by tailoring the plasma type and parameters: metallic nano-clusters can be deposited on a variety of substrates for catalytic purposes (e.g., lowtemperature fuel cell for transportation or autonomous power units) but plasmabased processes at the nano-scale usually require low pressure plasmas which are costly compared to near-atmospheric pressure plasmas [17-19]. For example, plasma-enhanced chemical vapor deposition is a common method sometime combined with annealing steps to control electrochemical and/or catalytic processes for the fabrication of energy conversion devices such as solar cells and fuel cells [20] and to manipulate local electronic structures (i.e., N-doping) for energy storage devices such as microbatteries and supercapacitors [21].

Judicious sequences of cold plasma processes (i.e., plasma-enhanced chemical vapor deposition, plasma-electrochemical deposition, low pressure radiofrequency plasmas, microdischarges, plasma-liquid interface systems) can also provide innovative routes for the synthesis of nanoparticles and nanomaterials yielding increased active surface areas, attachment of functional groups on surfaces or other material properties [18, 22-25, 38].

A key driver of Twenty first century science, technology and world economy will be "access to space," based on 55 years of success in the development of satellites starting with the first $83 \mathrm{~kg}$ satellite (Sputnik in 1957), the first meteorology satellite (Titos in 1960) and the first telecommunications satellite (Telstar in 1962). The largest human-made spacecraft is the International Space Station, which was initiated in 1983, now weighs 400 tons and cost over 100 billion US dollars. The NASA Voyager 1 exploratory spacecraft, which was launched in 1977, has now traveled in space further than any other human-made craft and has left our solar system. Our daily life strongly relies on successful operation of hundreds of satellites placed on Low Earth Orbits and Geostationary Orbits. Political, economical and military interest drive an unprecedented number of countries to invest in space activities leading to increasing concern for collisions between satellites and space debris. New regulations are being implemented such as a required placement of satellites on graveyard orbits at the end of their lifetime, an enhanced control of toxic propellants used in chemical propulsion engines such as hydrazine or of expensive propellant used in electric propulsion such as xenon (permanently depleting the small reserve available on Earth). The development of new plasma thruster technologies which 
operate at very low pressure for various types of propellants and could use normally unused residual liquids and gases to provide additional velocity increment would, for example, allow the nominal mission to be extended to the "last drop of fuel" with the capability to still be able to de-orbit a spacecraft. In addition, with the emergence of CubeSat satellites and a variety of new remote sensing technologies providing cheaper access to space, there is increasing demand for low-cost small propulsion systems which can be operated with "greener" or cheaper propellants.

\section{POPULATION HEALTH}

The chemical properties of plasma discharges can be used to produce active species with some very simple processes in place. Population health starts with clean water. Drinking water disinfection using ozone, a powerful oxidizer, is one of the oldest plasma processes usually involving a corona discharge in pure oxygen or air. Although ozone is an air pollutant (harmful under prolonged exposure), it is widely used to kill microorganisms in water (i.e., swimming pools), on food or on contact surfaces (laundries in hospitals, health care facilities) and also in the preparation of pharmaceuticals.

The thermal properties of plasmas have also been extensively used in medicine for cauterization: the plasma scalpel is a "thermal knife" which generates almost no blood loss and allows enhanced precision and simultaneous division and coagulation with less damage (area and intensity) to the surrounding tissues compared to a steel or electrosurgical scalpel. Cauterization occurs on tissue in direct contact with the argon plasma jet with limited heat transferred to the surrounding tissues. This type of jet can also be used for coagulation of bleeding lesions. In addition to this physical interaction between plasma and tissue, therapeutic effects related to numerous components of plasma, such as reactive species (O, NOx, etc.), charged particles, electric fields and even UV light may occur [26]. To this effect a new area of "Plasma Medicine" in close relation with "Microplasmas" is emerging which is related to the development of non-thermal atmospheric-pressure plasma sources (the gas temperature is much lower than the electron temperature) aimed at triggering complex sequence of biological responses in tissues and cells with possible future applications in dental cavities, skin diseases, wound healing and even cancer treatment. Challenges will be overcome via a better understanding of the complex mechanisms of what plasma does to the background medium and interface via external parameters such as frequency, voltage and waveform of the electrical pulses.

There are many reviews which highlight the current challenges and breakthroughs in Plasma Medicine [27, 28]; this field of research is based on pioneer research work carried out early in this century [29, 30] and has grown into a cluster of very active sub-fields: mechanisms of plasma in cancer therapy [31], plasma sources used in medicine [32], plasma treatment of stem cells [33], development of tissue models [34] to cite a few.

Other fast developing areas of relevance to plasma-based technology for medical applications include biomedical transducers, microfluidics, medical implants, microsurgical tools and tissue engineering $[35,36]$. Low pressure plasma polymer processes are also increasingly used for the deposition of thin organic coatings with a biological response associated with the presence of functional groups [37]. Overlap between various growing areas of plasma processing science exists ("Liquid plasmas," "Plasma polymers," "Bio-MEMS") as the interaction of plasma with living system lies at the frontier of plasma science (physics and engineering), chemistry and biology.

Whether for convenience (esthetic and art) or necessity (food and shelter), a growing population means growing needs and this is bound to dictate the direction of some future developments. Examples include the deposition of inorganic barrier coatings $(\mathrm{SiOx})$ on a variety of substrates (nylon, PET) for food packaging of liquids or complete meals, the treatment of protective clothing and even the deposition of esthetically pleasing coatings.

\section{CONCLUSION}

In the area of plasma physics, there is overall a need for, rather than an abundance of, available data spanning from the atomic to the macroscopic and intergalactic scale.
One iconic grand challenge for cold plasma physics is the unexplained acceleration of the Solar Wind. It clearly shows the necessity to further develop our understanding of complex mechanisms occurring in space, and somewhat harnessed on Earth, by the use of novel approaches to experimental, modeling and theoretical work. This should be facilitated by the development of computational power, of new infrastructure and diagnostic tools and the ability to link expertise in various areas via platforms on the World Wide Web.

Over the past few decades the development of low-temperature plasmas and their applications has directly impacted on our daily lives through major development in electronic, communication and lighting devices to name a few. There is a critical need for further development in most technologies as the Earth's fragile equilibrium is increasingly threatened by human activities and needs. Many challenges relate to the cross-disciplinary nature of most developments and a few key themes have been reviewed.

Low-temperature plasma physics has already demonstrated its ability to contribute to a wide range of areas of science as it often lies at the "boundaries" between these areas. These boundaries and their fluctuations are at the heart of inter-connectivity between many traditional and emerging areas of science. The overall challenge perhaps lies in awareness of the connectivity to ensure long term creativity and readiness for changes ahead in society, industry and science.

\section{REFERENCES}

1. Parker EN. Reflections on macrophysics and the sun. Solar Phys. (1997) 176:219-47. doi: 10.1023/A:1005072231043

2. Lowke JJ. Plasma predictions: past, present and future. Plasma Source Sci Technol. (2013) 22:023002. doi: 10.1088/0963-0252/22/2/023002

3. Pfalzner S. An Introduction to Inertial Confinement Fusion. New York, NY: CRC press Taylor and Francis Group (2006).

4. Gibson EG. The Quiet Sun. Washington, DC: National Aeronautics and Space Administration (1972).

5. Schindler K. Laboratory experiments related to the solar wind and the magnetosphere. Rev Geophys. (1969) 7:51-75. doi: 10.1029/RG007i001p00051

6. Warren HP, Mauel ME. Observation of chaotic particle transport induced by driftresonant fluctuations in a magnetic dipole 
field. Phys Rev Lett. (1994) 74:1351-4. doi: 10.1103/PhysRevLett.74.1351

7. Carlqvist P, Alfven H. Energy source of the solar wind. Astrophys Space Sci. (1980) 71:203-9. doi: 10.1007/BF00646918

8. Tu CY, Zhou C, Marsch E, Xia LD, Zhao L, Wang JX, et al. Solar wind origin in coronal funnels. Science (2005) 308:519. doi: 10.1126/science.1109447

9. Koepke ME. Interrelated laboratory and space plasma experiments. Rev Geophys. (2008) 46:RG3001. doi: 10.1029/2005RG000168

10. Von Steiger R. Space physics-grand challenges for the 21 st century. Front Phys. (2013) 1:6. doi: 10.3389/fphy.2013.00006

11. Pecseli H. Waves and Oscillations in Plasmas. New York, NY: CRC press Taylor \& Francis Group (2012).

12. Samukawa S, Hori M, Rauf S, Tachibana K, Bruggeman P, Kroesen G, et al. The 2012 plasma roadmap. J Phys D Appl Phys. (2012) 45:253001. doi: 10.1088/0022-3727/45/25/253001

13. Lieberman MA, Lichtenberg AJ. Principles of Plasma Discharges and Materials Processing. New York, NY: Wiley-Interscience (1994).

14. Donnelly VM, Kornblit A. Plasma etching: yesterday, today, and tomorrow. J Vacuum Sci Technol A. (2013) 31:050825. doi: 10.1116/1.4819316

15. Malka V, Faure J, Gauduel YA, Lefebvre E, Rousse A, Phuoc KT. Principles and applications of compact laser-plasma accelerators. Nat Phys. (2008) 4:447. doi: $10.1038 /$ nphys 966

16. Hooker SM. Developments in laser-driven plasma accelerators. Nat Photon. (2013) 7:775. doi: 10.1038/nphoton.2013.234

17. Caillard A, Charles C, Boswell R, Brault P, Coutanceau C. Plasma based platinum nanoaggregates deposited on carbon nanofibers improve fuel cell efficiency. Appl Phys Lett. (2007) 90:223119. doi: 10.1063/1.2745210

18. Hatakeyama R, Kato T, Li Y, Kaneko T. Plasma processing based synthesis of functional Nanocarbons. Plasma Chem Plasma Proc. (2014) 34:377-402. doi: 10.1007/s11090-0149547-z

19. Kakiuchi H, Ohmi H, Yasutake K. Atmosphericpressure low-temperature plasma processes for thin film deposition. J Vacuum Sci Technol A. (2014) 32:030801. doi: 10.1116/1.4828369

20. Brault P. Plasma deposition of catalytic thin films: experiments, applications, molecular modeling. Surf Coat Technol. (2011) 205:S15-S23. doi: 10.1016/i.surfcoat.2011.01.052

21. Jeong HM, Lee JW, Shin WH, Choi YJ, Shin HJ, Kang JK, et al. Nitrogen-doped graphene for high-performance ultracapacitors and the importance of nitrogen-doped sites at basal planes. NanoLetters (2011) 11:2472-7. doi: $10.1021 / \mathrm{nl} 2009058$

22. Richmonds C, Mohan Sankaran R. Plasmaliquid electrochemistry: rapid synthesis of colloidal metal nanoparticles by microplasma reduction of aqueous cations. Appl Phys Lett. (2008) 93:131501. doi: 10.1063/ 1.2988283

23. Meiss SA, Rohnke M, Kienle L, Zein EI Abedin $S$, Endres F, Janek J. Employing plasmas as gaseous electrodes at the free surface of ionic liquids: deposition of nanocrystalline silver particles. Chem Phys Chem. (2007) 8:50-3. doi: 10.1002/cphc.200600582

24. Mangolini L, Thimsen E, Kortshagen U. Highyield plasma synthesis of luminescent silicon Nanocrystals. NanoLetters (2005) 5:655-9. doi: $10.1021 / \mathrm{nl} 050066 \mathrm{y}$

25. Chiang WH, Mohan Sankaran R. Linking catalyst composition to chirality distributions of as-grown single-walled carbon nanotubes by tuning NixFel-x nanoparticles. Nat Mater. (2009) 8:882-6. doi: 10.1038/nmat2531

26. Graves DB. The emerging role of reactive oxygen and nitrogen species in redox biology and some implications for plasma applications to medicine and biology. I Phys D Appl Phys. (2012) 45:263001. doi: 10.1088/0022$3727 / 45 / 26 / 263001$

27. von Woedtke TH, Reuter S, Masur K, Weltmann K-D. Plasmas for medicine. Phys Rep Rev Sect Phys Lett. (2013) 530:291-320. doi: 10.1016/j. physrep.2013.05.005

28. Kong MG, Kroesen G, Morfill G, Nosenko T, Shimizu T, Van Dijk J, et al. Plasma medicine: an introductory review. New J Phys. (2009) 11:115012. doi: 10.1088/1367-2630/11/ $11 / 115012$

29. Stoffels E, Kieft IE, Sladek REJ. Superficial treatment of mammalian cells using plasma needle. J Phys D Appl Phys. (2003) 36:2908-13. doi: 10.1088/0022-3727/36/23/007

30. Fridman G, Peddinghaus $M$, Balasubramanian M, Ayan H, Fridman A, Gutsol A, et al. Blood coagulation and living tissue sterilization by floating-electrode dielectric barrier discharge in air. Plasma Chem Plasma Process. (2006) 26:425-42. doi: 10.1007/s11090-0069024-4

31. Keidar M, Shashurin A, Volotskova O, Stepp MA, Srinivasan P, Sandler A, et al. Cold atmospheric plasma in cancer therapy. Phys Plasmas (2013) 20:057101. doi: 10.1063/1.4801516
32. Weltmann KD, Polak M, Masur K, Von Woedtke $\mathrm{T}$, Winter J, Reuter S. Plasmas processes and plasma sources in medicine. Contrib Plasma Phys. (2012) 52:644-54. doi: 10.1002/ctpp.2012 10061

33. Miletic M, Mojsilovic S, Okic Dordevic I, Maletic D, Puac N, Lazovic S, et al. Effect of non-thermal atmospheric plasma on human periodontal ligament mesenchimal stem cells. J Phys D Appl Phys. (2013) 46:345401. doi: 10.1088/0022-3727/46/34/345401

34. Szili EJ, Bradley JW, Short RD. A 'tissue model' to study the plasma delivery of reactive oxygen species. J Phys D Appl Phys. (2014) 47:152002. doi: 10.1088/0022-3727/47/15/152002

35. Langer R, Tirrell DA. Designing materials for biology and medicine. Nature (2004) 428:487-92. doi: 10.1038/nature02388

36. Nguyen NT, Shaegh SAM, Kashaninejad N, Phan D-T. Design, fabrication and characterization of drug delivery systems based on lab-on-a-chip technology. Adv Drug Deliv Rev. (2013) 65:140319. doi: 10.1016/j.addr.2013.05.008

37. Wertheimer MR. Plasma processing and polymers: a personal perspective. Plasma Chem Plasma Process. (2014) 34:363-76. doi: 10.1007/s11090-013-9491-3

38. Kaneko T, Takahashi S, Hatakeyama R. Control of nanoparticle synthesis using physical and chemical dynamics of gas-liquid interfacial non-equilibrium plasmas. Plasma Phys Control Fusion (2012) 54:124027. doi: 10.1088/0741$3335 / 54 / 12 / 124027$

Conflict of Interest Statement: The author declares that the research was conducted in the absence of any commercial or financial relationships that could be construed as a potential conflict of interest.

Received: 14 May 2014; accepted: 11 June 2014; published online: 27 June 2014.

Citation: Charles C (2014) Grand challenges in lowtemperature plasma physics. Front. Phys. 2:39. doi: 10.3389/fphy.2014.00039

This article was submitted to Plasma Physics, a section of the journal Frontiers in Physics.

Copyright (c) 2014 Charles. This is an open-access article distributed under the terms of the Creative Commons Attribution License (CC BY). The use, distribution or reproduction in other forums is permitted provided the original author(s) or licensor are credited and that the original publication in this journal is cited, in accordance with accepted academic practice. No use, distribution or reproduction is permitted which does not comply with these terms. 